\title{
Central Hyperthermia Treated With Baclofen for Patient With Pontine Hemorrhage
}

\author{
Hyun Cheol Lee, $\mathrm{MD}^{1}$, Jong Moon Kim, MD ${ }^{1}$, Jae Kulk Lim, MD ${ }^{1}$, \\ Yoon Sik Jo, MD², Shin Kyoung Kim, $\mathrm{MD}^{1}$ \\ Departments of ${ }^{1}$ Rehabilitation Medicine and ${ }^{2}$ Neurology, Konkuk University School of Medicine, Chungju, Korea
}

\begin{abstract}
Central hyperthermia is a very rare disease; however, once it happens, it is associated with a poor prognosis and high mortality for patients with severe brainstem strokes. Following a pontine hemorrhage, a 46 -yearsold female developed prolonged hyperthermia. Work-ups to the fever gave no significant clues for the origin of fever, and hyperthermia did not respond to any empirical antibiotics or antipyretic agents. The patient's body temperature still fluctuated in a range of $37.5^{\circ} \mathrm{C}$ to $39.2^{\circ} \mathrm{C}$. Considering the lesion of hemorrhage, we suspected central hyperthermia rather than infectious diseases. We started with baclofen administration at a dose of $30 \mathrm{mg} /$ day. The body temperature changed to a range of $36.6^{\circ} \mathrm{C}$ to $38.2^{\circ} \mathrm{C}$. We raised the dose of baclofen to $60 \mathrm{mg} / \mathrm{day}$. The patient's body temperature finally dropped to a normal range. Central hyperthermia, caused by failures of thermoregulatory pathways in brainstem, following the pontine hemorrhage rarely occurs. Baclofen can be used to treat suspected central hyperthermia in a patient with pontine hemorrhage.
\end{abstract}

Keywords Fever, Pons, Hemorrhage, Baclofen

\section{INTRODUCTION}

Prolonged central hyperthermia following pontine hemorrhage is a very rare disease, caused by failures of thermoregulatory pathways at the brainstem. Although central hyperthermia has been known for a long time, our knowledge remains limited.

Central hyperthermia is usually associated with a high body temperature that does not respond to common

Received September 20, 2012; Accepted November 19, 2012

Corresponding author: Shin Kyoung Kim

Department of Rehabilitation Medicine, Konkuk University School of Medicine, 268 Chungwon-daero, Chungju 380-150, Korea

Tel: +82-43-840-8890, Fax: +82-43-851-3402, E-mail: kimnerve@hanmail.net

(c) This is an open-access article distributed under the terms of the Creative Commons Attribution Non-Commercial License (http://creativecommons. org/licenses/by-nc/3.0) which permits unrestricted noncommercial use, distribution, and reproduction in any medium, provided the original work is properly cited.

Copyright (C) 2014 by Korean Academy of Rehabilitation Medicine antipyretic treatments [1]. According to some literature, high fever $\left(>39^{\circ} \mathrm{C}\right)$ with tachycardia $(>110$ beat $/ \mathrm{min})$ developed within 24 hours from the onset of stroke is correlated with high risks of death $[2,3]$. Furthermore, if patients survived from the above situation, most survivors remained in a severely disabled state.

In this respect, early diagnosis and treatment of central hyperthermia is critical. However, in most cases, it is difficult to diagnose early because of high mortality rates due to complications, such as sudden cardiac deaths, acute renal failures, rhabdomyolysis, etc., induced by high fevers and the time duration required to perform numerous tests for exclusion of infected sources.

A patient who had hyperthermia for more than a month without specific fever focus was being transferred to our department. We diagnosed her with prolonged central hyperthermia considering injured lesion. She was treated with baclofen and thus, we reported the case and pro- 
vided relative literature reviews.

\section{CASE REPORT}

Following pontine hemorrhage, a 46-years-old female developed prolonged hyperthermia. At the day of admission, she visited our emergency department with symptoms of a sudden headache, weakness on both upper extremities, and mental changes. She did not have specific past histories, such as diabetic mellitus, hypertension, pulmonary tuberculosis, hepatitis B, and so on. There were no preceding infections or fevers at least 1 week prior to the onset of pontine hemorrhage.

Brain computed tomography (CT) was conducted and hemorrhage was observed throughout lower midbrain to pons (Fig. 1). She took the non-surgical treatment at neurosurgeon department. One day after the admission, fever above $38^{\circ} \mathrm{C}$ and intermittent tachycardia ( $>110$ beat/min) developed approximately 1-2 times a day. Whole work-ups to the fever, including laboratory tests (complete blood count, white blood cell differ-count, C-reactive protein, erythrocyte sedimentation rate, electrolyte, urinalysis, urine microscopy, aspartate aminotransferase, alanine aminotransferase, gamma-glutamyl transpeptidase, total bilirubin, blood urea nitrogen/creatinine, microbial culture on blood, sputum, urine and cerebrospinal fluid analysis, etc.) and radiologic study (chest X-ray and chest CT to rule out pneumonia, paranasal sinus water's view to rule out sinusitis), showed no significant clues for the origin of fever. Despite the consultation to department of infectious diseases, the origin of fever was still ambiguous and empirical antibiotic treatments were performed, but the fever did not respond to any antibiotics (cefbuperazone+clindamycin, amoxicillin+clavulanate, ceftazidime, meropenem) or antipyretics (acetaminophen, non-steroidal anti inflammatory drugs, aspirin lysinate, etc.). Suspicions of drug fevers, antiepileptic agents (valproic acids) and any nonessential drugs (neurostimulators, such as phenytoin, donepezil, acetyl-L-carnitine, antipyretics, etc.) were then discontinued. But, the body temperature was still fluctuating in a range of $37.5^{\circ} \mathrm{C}$ to $39.2^{\circ} \mathrm{C}$ and tepid massage was the only way to control the fever. After 40 days from admission, she was transferred to the department of rehabilitation medicine. Intermittent fever over $38^{\circ} \mathrm{C}$ and tachycardia ( $>110$ beat/min) was still ongoing. Whole work-up of fever was tested again, and still the results indicated no clues for the origin of fever. Since then, we suspected the possibility of non-infectious diseases so we searched literatures related to fevers of unknown origin in patients diagnosed with intracranial hemorrhages. Assuming the lesion of hemorrhage, central hyperthermia was strongly suspected and there was a case report of central hyperthermia treated by baclofen [4], thus, we started baclofen oral administration at a dose of $30 \mathrm{mg} /$ day after 44 days from admission. Then, the body temperatures tended to subside, however, intermittent fever sustained at a range of $36.6^{\circ} \mathrm{C}$ to $38.2^{\circ} \mathrm{C}$. To further drop the body temperature, we raised the dose of baclofen to $60 \mathrm{mg} /$ day. Finally, after 47 days from admission, the
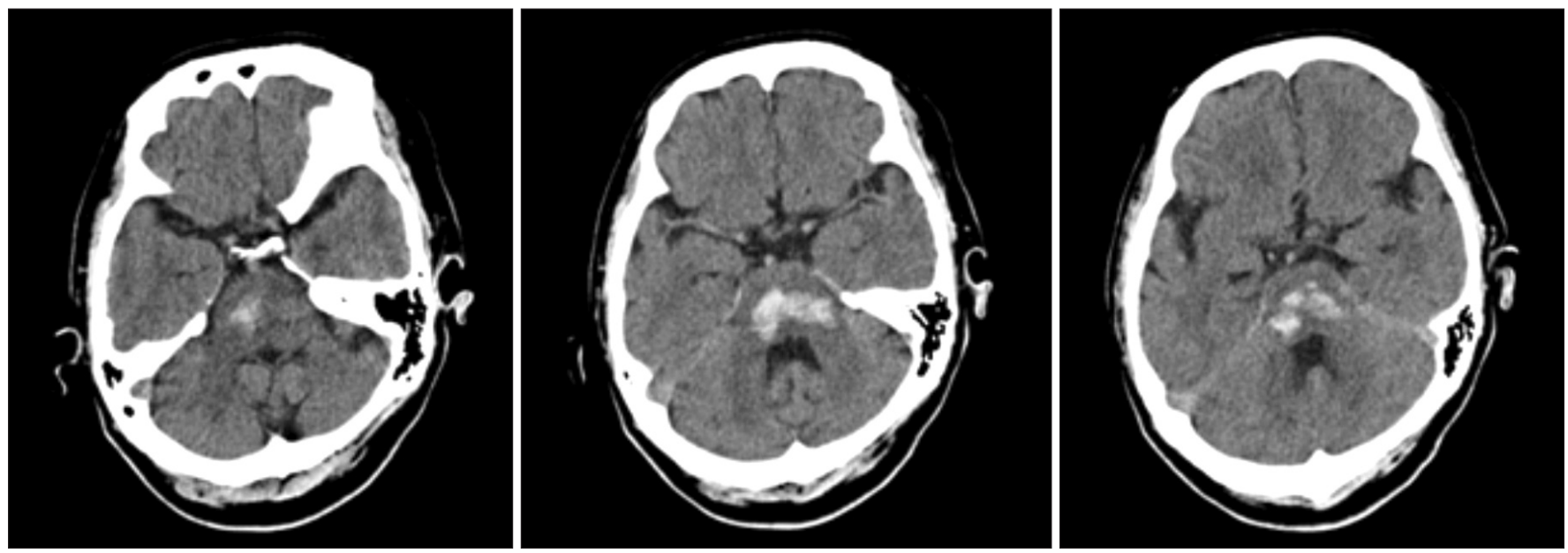

Fig. 1. Computed tomography demonstrated hemorrhage in brainstem and middle cerebellar peduncle which are mainly posterior pons and the largest diameter is $29 \mathrm{~mm}$. 
body temperature dropped to a normal range and was maintained stably (Fig. 2). Until now, she is being administered $60 \mathrm{mg} /$ day of baclofen and her basal body temperature is being maintained within normal limits. She is undergoing comprehensive rehabilitation for the sequelae of pontine hemorrhages without any specific side effects of baclofen.

\section{DISCUSSION}

Central hyperthermia is likely a result of direct pontine destruction or indirect compression. Central hyperthermia is characterized by an unchanged setting of the thermoregulatory center so antipyretics have no effects on central hyperthermia.

Diagnosis of central hyperthermia must meet the following criteria: 1) no preceding infections or fevers at least 1 week prior to stroke onset, 2) high fever $\left(>39^{\circ} \mathrm{C}\right)$ developing within 24 hours after onset of stroke, and 3) negative work-ups for fever of infectious origin [2]. But the underlying pathophysiology of this condition is unknown.

Body temperature is strictly controlled in humans at approximately $37^{\circ} \mathrm{C}$. Several physiologic mechanisms are involved in temperature homeostasis, all of which are coordinated through the hypothalamus [4]. The central pathway for control of thermoregulatory thermogenesis and innervations of brown adipose tissue (BAT) is complex [5]. BAT is an important effector organ for non-shivering thermogenesis in the defense against cold [4]. Pre-

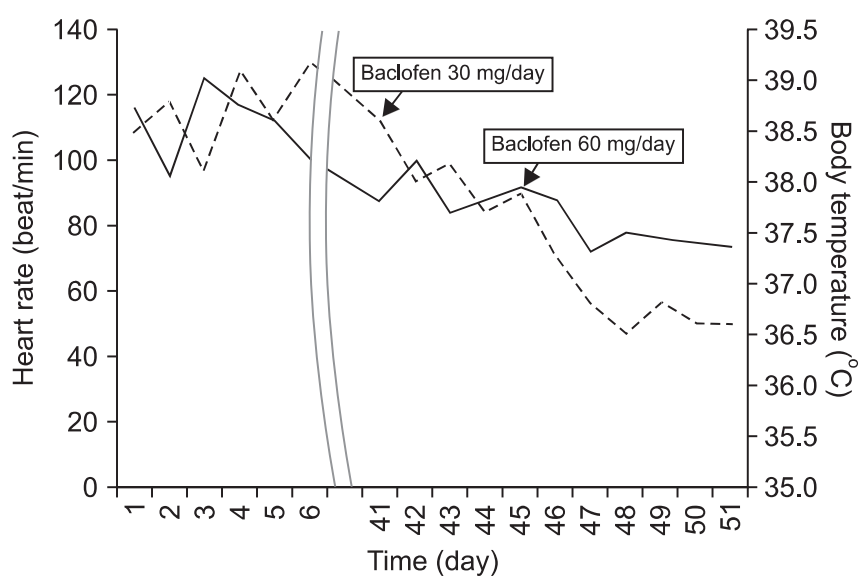

Fig. 2. Fever patterns of prolonged central hyperthermia in a pontine hemorrhage. optic chiasma/anterior hypothalamic nuclei are accepted as the center for body temperature control. Cooling of this area activates BAT [6], whereas warming suppresses activation of BAT and non-shivering thermogenesis [7]. The efferent signals from the preoptic chiasma/anterior hypothalamic nuclei are the inhibitor of gammaaminobutyric acids (GABA) to reach ventromedial hypothalamic nucleus [8]. The signal from the ventromedial hypothalamic nucleus reached the raphe nuclei through the lower midbrain and release GABA in this area $[9,10]$. The thermoregulatory signal is connected to the sympathetic chain. The sympathetic chain controls the BATs for non-shivering thermogenesis.

We hypothesized that baclofen had an effect on central hyperthermia for patients with pontine hemorrhages. Baclofen, a GABA agonist, functioned as inhibitory signals directly acting on the raphe nuclei to suppress BAT activation, which in turn suppresses the body temperatures [4]. Thus, baclofen may control central hyperthermia by replacing neurotransmitters (GABA, glutamate) which were blocked due to the location of hemorrhage.

The present case provided us guidance for understanding thermoregulatory dysfunction in patients with brainstem hemorrhage. In the case of pontine strokes, we should include prolonged central hyperthermia as possible causes of fever and consider the administration of baclofen.

\section{CONFLICT OF INTEREST}

No potential conflict of interest relevant to this article was reported.

\section{REFERENCES}

1. Morales-Ortiz A, Jimenez-Pascual M, Perez-Vicente JA, Monge-Arguiles A, Bautista-Prados J. Fever of central origin during stroke. Rev Neurol 2001;32:1111-4.

2. Sung CY, Lee TH, Chu NS. Central hyperthermia in acute stroke. Eur Neurol 2009;62:86-92.

3. Wijdicks EF, St Louis E. Clinical profiles predictive of outcome in pontine hemorrhage. Neurology 1997;49:1342-6.

4. Huang YS, Hsiao MC, Lee M, Huang YC, Lee JD. Baclofen successfully abolished prolonged central hyperthermia in a patient with basilar artery occlusion. 
Acta Neurol Taiwan 2009;18:118-22.

5. Cannon B, Nedergaard J. Brown adipose tissue: function and physiological significance. Physiol Rev 2004;84:277-359.

6. Imai-Matsumura K, Nakayama T. The central efferent mechanism of brown adipose tissue thermogenesis induced by preoptic cooling. Can J Physiol Pharmacol 1987;65:1299-303.

7. Boulant JA. Role of the preoptic-anterior hypothalamus in thermoregulation and fever. Clin Infect Dis 2000;31 Suppl 5:S157-61.

8. Amir S, Schiavetto A. Injection of prostaglandin E2 into the anterior hypothalamic preoptic area activates brown adipose tissue thermogenesis in the rat. Brain Res 1990;528:138-42.

9. Shibata M, Iriki M, Arita J, Kiyohara T, Nakashima T, Miyata S, et al. Procaine microinjection into the lower midbrain increases brown fat and body temperatures in anesthetized rats. Brain Res 1996;716:171-9.

10. Morrison SF, Sved AF, Passerin AM. GABA-mediated inhibition of raphe pallidus neurons regulates sympathetic outflow to brown adipose tissue. Am J Physiol 1999;276(2 Pt 2):R290-7. 